\title{
Factores de riesgo asociados con la aparición de conductas suicidas en adolescentes
}

\author{
Risk Factors Associated with the Appearance of Suicidal \\ Behaviors in Adolescents
}

\section{Fatores de risco associados ao aparecimento de comportamentos suicidas nos adolescentes}

\author{
Claudia Patricia Serrano-Ruiz, FT., MSc. * \\ Julie Alexandra Olave-Chaves, Psi., MSc. **
}

\begin{abstract}
Resumen
Introducción: El suicidio en adolescentes es un problema de salud pública dada su elevada incidencia y el gran impacto que este genera no solo a nivel individual, sino familiar y social. Existen algunos factores que, de acuerdo con la literatura, han sido asociados con el desarrollo de conductas suicidas en los adolescentes, sin embargo, su descripción se ha realizado de manera aislada, desconociendo la multicausalidad del problema. Objetivo: Revisar en la literatura científica los factores personales, familiares y sociales asociados con la aparición de conductas suicidas en adolescentes. Metodología: Se efectuó una búsqueda y análisis de la información empleando los descriptores adolescentes, suicidio, ideación suicida, factores de riesgo, atención primaria en salud y salud mental. Dicha búsqueda
\end{abstract}

se realizó en los buscadores Google, Google académico y en las bases de datos ScienceDirect, PubMed, ProQuest, Scielo, Redalyc. Resultados: Se encontraron factores de riesgo para la aparición de conductas suicidas en adolescentes relacionados con el género, el nivel educativo y socioeconómico, las relaciones familiares y las redes de apoyo social, entre otros. Conclusiones: La oportuna detección de los factores de riesgo podría aportar en gran medida al diseño e implementación de programas de prevención más integrales y eficientes frente al suicidio de adolescentes. [Serrano-Ruiz CP, Olave-Chaves JA. Factores de riesgo asociados con la aparición de conductas suicidas en adolescentes. MedUNAB 2017; 20(2): 139-147].

Palabras clave: Suicidio; Factores de Riesgo; Adolescente; Asociación; Atención Primaria de Salud; Salud Mental.

* Fisioterapeuta, magíster en Salud Pública, docente seminario Salud Mental y Atención Primaria en Salud, Programa de Psicología, Universidad Cooperativa de Colombia sede Bucaramanga, Santander, Colombia.

** Psicóloga, magíster en Salud Pública, jefe del Programa de Psicología, Universidad Cooperativa de Colombia, Bucaramanga, Santander, Colombia. 


\section{Abstract}

Introduction: Suicide in adolescents is a public health problem given its high incidence and the great impact it generates not only at an individual level, but also at a family and social level. There are some factors that, according to literature, have been associated with the development of suicidal behavior in adolescents; however, their descriptions have been made in isolation, ignoring the multicausality of the problem. Objective: To review in the scientific literature the personal, family and social factors associated with the appearance of suicidal behaviors in adolescents. Methodology: A search and an analysis of the information was carried out using some descriptors such as adolescent, suicide, suicidal ideation, risk factors, primary health care and mental health. This search was carried out in search engines like Google, Google Scholar and in the databases ScienceDirect, PubMed, ProQuest, Scielo and Redalyc. Results: Some risk factors were found related to the appearance of suicidal behaviors in adolescents which are associated to gender, educational and socioeconomic level, family relationships and social support networks, among others. Conclusions: Timely detection of risk factors could greatly contribute to the design and implementation of more comprehensive and efficient prevention programs against adolescent suicide. [SerranoRuiz CP, Olave-Chaves JA. Risk Factors Associated with the Appearance of Suicidal Behaviors in Adolescents. MedUNAB 2017; 20(2): 139-147].

Keywords: Suicide; Risk Factors; Adolescent; Association; Primary Health Care; Mental Health.

\section{Introducción}

La conducta suicida es entendida como "todo acto por el que un individuo se causa a sí mismo una lesión o un daño, cualquiera sea el grado de la intención letal o de conocimiento del verdadero móvil" (1). Comprende la ideación suicida, el intento de suicidio y el suicidio consumado. La ideación suicida se entiende como la presencia de ideas o deseos suicidas, es decir, es el conjunto de pensamientos sobre el cese de la propia vida. Por su parte, el intento de suicidio incluye las tentativas o actos autolesivos y deliberados con los que se busca morir y, finalmente, el suicidio consumado es el resultado exitoso del intento de acabar con la vida $(1,2)$. La presencia de la conducta suicida en los adolescentes es percibida como " $l a$ manifestación o resultante de la carencia de mecanismos adaptativos del sujeto al medio, generada por una situación de conflicto actual o permanente, que causa un estado de tensión emocional"'(3).

La adolescencia es una etapa de cambios físicos y psicológicos atribuidos especialmente a modificaciones en los procesos hormonales, que marcan un periodo de transición entre la niñez y la edad adulta (4). Durante la adolescencia se generan cambios psicosociales asociados con la adaptación e integración de una nueva estructura

\section{Resumo}

Introdução: O suicídio nos adolescentes é um problema de saúde pública porque sua alta incidência e o grande impacto que gera não só a nível individual, mas também a nível familiar e social. Existem alguns fatores que, de acordo com a literatura, foram associados ao desenvolvimento dos comportamentos suicidas nos adolescentes, no entanto, sua descrição foi realizada de forma isolada, ignorando a multi-causalidade do problema. Objetivo: Revisar na literatura científica os fatores pessoais, familiares e sociais associados ao aparecimento dos comportamentos suicidas nos adolescentes. Metodologia: Realizamos uma pesquisa e análise da informação usando os relatos dos adolescentes, do suicídio, da concepção suicida, dos fatores de risco, dos cuidados de saúde primários e de saúde mental. Esta pesquisa foi realizada em motores de busca do Google, Google acadêmico e nas bases de dados ScienceDirect, PubMed, ProQuest, Scielo, Redalyc. Resultados: Os fatores de risco encontrados no aparecimento de comportamentos suicidas em adolescentes, estão relacionados com o gênero, o nível de educação e ao contexto socioeconómico, às relações familiares e às redes de apoio social, entre outros. Conclusões: Detectar os fatores de risco a tempo poderia contribuir grandemente na elaboração do projeto e implementação de programas de prevenção mais completos e eficientes contra o suicídio do adolescente. [Serrano-Ruiz CP, Olave-Chaves JA. Fatores de risco associados ao aparecimento de comportamentos suicidas nos adolescentes.MedUNAB 2017; 20(2): 139-147].

Palavras-chave: Suicídio; Fatores de Risco; Adolescente; Associação; Atenção Primária à Saúde; Saúde Mental.

corporal, la reestructuración de la identidad y la anticipación de un futuro a través de la construcción de un proyecto de vida (5). Cuando los adolescentes no cuentan con los mecanismos de afrontamiento adecuados para sobreponerse a estos cambios y a las nuevas exigencias de su medio, pueden llegar a acudir a conductas suicidas como vías para resolver sus conflictos y alivianar las diversas presiones familiares y sociales, de sus iguales y de sí mismos (6). Puesto que la adolescencia es una época de exploración de la identidad sexual y de conformación de relaciones sociales, la necesidad de independencia contradice a menudo las reglas y expectativas que otras personas establecen. El suicidio y el intento de consumarlo, componen las expresiones más dramáticas del fracaso de los adolescentes al enfrentarse con este nuevo mundo (7).

La prevención del suicidio es apremiante, por lo que identificar los factores de riesgo para el desarrollo de conductas suicidas podría contribuir a la disminución de esta problemática $(8,9)$. Existen algunos factores que han sido identificados en la literatura como asociados con el desarrollo de conductas suicidas en esta población; sin embargo, su descripción se ha dado de manera aislada, sin permitir hacer evidente el origen multicausal de esta problemática. Dentro de los factores descritos se encuentran aspectos relacionados con el género, el estado de salud mental, la composición familiar, la discriminación social y 
la carencia de redes de apoyo, entre otros $(4,6,10-12)$. De acuerdo con esto, se puede determinar que los factores de riesgo para la aparición de la conducta suicida son diversos e involucran aspectos relacionados no sólo con el individuo sino también con el contexto que lo rodea.

La presente revisión tiene como objetivo identificar, con base en la revisión de la literatura científica, los principales factores de orden personal, familiar y social que han sido asociados con la aparición de conductas suicidas en adolescentes. Sin embargo, para entender mejor la importancia de profundizar en esta temática es necesario realizar una breve descripción inicial de los aspectos epidemiológicos más relevantes de esta problemática de salud pública, tanto en el ámbito mundial como nacional.

\section{Epidemiología de la conducta suicida}

De acuerdo con la Organización Mundial de la Salud (OMS), las conductas suicidas constituyen un grave problema de salud pública porque estas defunciones inesperadas, predominantemente de jóvenes y de adultos de mediana edad, dan como resultado una enorme carga personal, familiar y social. En el ámbito mundial, cada año más de 800,000 personas se quitan la vida, lo que indica que posiblemente cada 40 segundos una persona se suicida; además, se estima que cada persona que consuma este acto ha realizado varios intentos previos de suicidio (1).

Los suicidios se presenta a cualquier edad; en particular, el suicidio es la segunda causa de defunción en el grupo etario de 15 a 29 años, a nivel mundial, y un $75 \%$ de estos suicidios ocurren en países de ingresos bajos y medianos. Actualmente, el suicidio es la segunda causa principal de muerte violenta en el mundo (1).

En Colombia el suicidio es la cuarta forma de violencia, con una tasa de mortalidad para el año 2013 de 3.8 por cada 100,000 habitantes (8). Según datos del Instituto Nacional de Medicina Legal y Ciencias Forenses (INMLCF), por cada mujer se quitan la vida cuatro 4 hombres. El Centro de Referencia Nacional sobre Violencia (CRNV) del INMLCF, a través del Sistema Nacional de Vigilancia Epidemiológica, reportó que en el año 2013 se realizaron 1,810 necropsias cuya forma de muerte correspondió con el suicidio. Los cinco departamentos reportados con las tasas más altas de suicidio fueron Amazonas (6.7) Meta (6.2) Quindío (5.9) Putumayo (5.9) y Antioquia (5.2) (8).

Según la distribución por grupos de edad, las tasas más altas de suicidio a nivel nacional se presentaron en los grupos etarios correspondientes a 18-19 años (6.7), seguidos por el grupo de 20-24 años (6.5), de 25-29 años (5.6), de 30-34 años (5.5) y en la población adulto mayor en el grupo correspondiente a los 70-74 años (5.4) (8).

El suicidio no suele priorizarse como un importante problema de salud pública, a pesar del gran impacto social, económico y familiar que este conlleva. Por otro lado, no obstante los grandes avances que se han hecho en los últimos años en la investigación relacionada con este tema, persisten aspectos que dificultan la prevención y el manejo del suicidio en adolescentes, tales como el estigma social alrededor del suicidio y la incapacidad de los sistemas y servicios de salud para atender la población en riesgo (1).

En relación con esto, Cifuentes menciona que los esfuerzos del Estado Colombiano por reducir estas cifras aún no muestran los resultados esperados; si bien es cierto que el Plan Decenal en Salud Pública 2012-2021 aborda esta temática, el énfasis se realiza especialmente en intervención de la población con trastornos mentales. Esto limita el manejo integral de esta problemática, ya que el suicidio ha sido descrito como un fenomeno multicausal y multifactorial y, por ende, requiere del trabajo articulado de diferentes sectores (8).

\section{Metodología}

Para el desarrollo de la presente revisión se estableció como pregunta orientadora ¿cuáles son los factores asociados con la aparición de conductas suicidas en adolescentes?. Así mismo, se definió operacionalmente el concepto adolescencia, considerándose como la etapa que transcurre entre los 11 y 19 años de edad, según lo descrito por la Organización Mundial de la Salud. Posteriormente, se realizó una búsqueda y análisis de los documentos empleando los términos DeCS: adolescentes, suicidio, ideación suicida, factores de riesgo, atención primaria en salud y salud mental, al igual que los operadores booleanos $y / o$. Dicha búsqueda se llevó a cabo en los buscadores google, google académico y en las bases de datos ScienceDirect, PubMed, ProQuest, Scielo, Redalyc, desde el 8 de mayo hasta el 19 de junio del 2016. En total se seleccionaron y revisaron 49 documentos que cumplieron con los siguientes criterios de inclusión: revisiones de tema, artículos originales o revisiones sistemáticas, páginas institucionales de entidades gubernamentales y libros escritos en idioma español o inglés. No se incluyeron documentos publicados con anterioridad al año 1990 o que no abordarán la problemática en el grupo adolescente. Posteriormente, se procedió a sintetizar la información encontrada en fichas técnicas que sirvieron de apoyo para la estructuración de la revisión de tema.

\section{Resultados}

\section{Factores personales asociados con la aparición de conductas suicidas}

Existen ciertos factores personales que han sido mencionados en numerosos estudios como posibles desencadenantes de conductas suicidas en los adolescentes. Dentro de estos se incluyen aspectos tales como la edad, el género y el estado civil, y otros relacionados con los rasgos 
de personalidad, la orientación sexual y las enfermedades físicas y mentales preexistentes.

En relación con el género la mayoría de los referentes coinciden en que las mujeres intentan suicidarse con una frecuencia mayor que los hombres; sin embargo, estos últimos consuman el suicidio en una proporción superior que ellas $(4,6,13-16)$.

Así mismo, el género es un aspecto que puede llegar a determinar los métodos de autoagresión empleados por los jóvenes al momento de incurrir en el intento suicida. Las mujeres hacen un mayor uso de psicofármacos (ansiolíticos y antidepresivos, etc.) y de fármacos de uso general (hormonas tiroideas, analgésicos, hierro, etc.), a los cuales tienen fácil acceso en sus hogares. Por su parte, los hombres reaccionan con alta inexpresividad emocional y agresividad, haciendo uso de métodos más letales como el ahorcamiento, el uso de armas de fuego y el salto de altura para conseguir con mayor certeza el suicidio $(6,13-16)$. Al parecer, conductas suicidas tales como la ideación suicida no están asociadas con el género (17).

Por otro lado, es posible que el bajo nivel socioeconómico y educativo, aunado al desempleo en la familia sean considerados factores de riesgo para el suicidio en los jóvenes (18). Así, un bajo nivel socioeconómico puede ser un factor de riesgo para el desarrollo de intentos de suicidio, especialmente si la situación de escasez es prolongada $(19,20)$. Adicionalmente, el bajo nivel socioeconómico puede dificultar el acceso a los servicios de salud, especialmente del orden mental, por lo cual, el manejo integral de diversas condiciones que llevan a conductas suicidas puede estar limitado (21).

En un estudio realizado por González y colaboradores se pudo encontrar que los adolescentes que presentaron ideación o intento suicida poseen una relación conflictiva con el padre y/o la madre y reportaron que sus padres discuten especialmente por problemas económicos (22). Por lo tanto, se puede inferir que los problemas económicos dentro de una familia pueden generar situaciones de estrés y violencia intrafamiliar, lo cual conduce a ideación suicida, conductas suicidas y suicidio consumado.

Los problemas de aprendizaje y el fracaso escolar también se han descrito como factores importantes para el desarrollo de conductas suicidas. En relación al nivel educativo, podría decirse que, de acuerdo con diversos autores, aún no se ha estudiado a profundidad este aspecto como factor de riesgo. No obstante, es posible que el bajo nivel educativo se relacione con las conductas suicidas en este grupo poblacional debido a que puede estar influenciado por factores como el escaso rendimiento académico y la deserción escolar, aspectos que pueden tener relación con la baja autoestima y con la discriminación, entre otros(19).

Así, los problemas en el colegio son considerados como un factor de riesgo para el suicidio en los adolescentes puesto que la inadaptación social, las dificultades de comunicación entre los jóvenes y la desaprobación de asignaturas generan conflictos que difícilmente son aceptados o asimilados por este grupo etario $(18,23)$. Así mismo, el suicidio también se puede presentar con mayor frecuencia en los preuniversitarios, o los recién graduados de secundaria durante el periodo de transición por el cual atraviesan (24).

Se ha determinado también que los adolescentes con conductas suicidas presentan ciertos rasgos de personalidad característicos tales como emotividad, poca estabilidad emocional, inseguridad, aprehensión, angustia, ansiedad y frustración, los cuales, en cierta medida, están relacionados con desequilibrios hormonales propios de la edad y no necesariamente con un desequilibrio de la personalidad $(25,26)$.

Es importante aclarar que, dentro de las conductas suicidas, la ideación suicida es la más frecuente en niños y adolescentes de ambos géneros, y esta no necesariamente se asocia con la presencia de rasgos o trastornos psicopatológicos. Esto se diferencia de los intentos de suicidio, que son menos frecuentes, pero que sí se asocian con mayor frecuencia a trastornos psicopatológicos, como trastornos de conducta disocial, depresión, trastorno bipolar y otros $(26,27)$. Es posible que se presenten algunas manifestaciones, resultado de la presencia de estas psicopatologías, tales como actitudes de escape, de venganza, altruismo, búsqueda de riesgo, tendencia a percibirse como perdedor, baja tolerancia a la frustración, dificultad para resolver conflictos, desesperanza, abandono y conductas autolesivas $(26,28)$. Uno de los factores de riesgo más importantes para la consumación de un suicidio es el intento suicida previo, el cual a su vez puede estar directamente ligado a la presencia de psicopatologías en los jóvenes (29-31).

Por otra parte, la conducta auto lesiva y la ideación suicida constituyen síntomas de riesgo de suicidio en la adolescencia, definiendo la conducta auto lesiva como la realización socialmente inaceptable y repetitiva de cortes, golpes y otras formas de autoagresión que causan lesiones físicas leves o moderadas (32). El comportamiento auto lesivo se encuentra tanto en población general como psiquiátrica y hay autores que proponen que se considere un síndrome por sí mismo $(33,34)$. Aunque el comportamiento auto lesivo es conceptualmente distinto del suicidio diversos estudios muestran una relación muy estrecha entre ambos, siendo las conductas autolesivas un claro factor de riesgo para las tentativas suicidas (35-37).

Algunos investigadores plantean un continuum entre la conducta auto lesiva y el suicidio, ya que ambos comparten un elemento esencial que es la auto inflicción de daño y únicamente se diferencian en la seriedad de la lesión causada $(38,39)$. Owens y colaboradores establecieron que un año después de un cuadro de autolesión el adolescente tiene cerca de un $2 \%$ de riesgo de suicidarse y, diez años después este riesgo aumenta a 5\% aproximadamente (29). Así 
mismo, se ha establecido una asociación existente entre las conductas auto lesivas (con y sin intención de muerte) y condiciones como la depresión y los desórdenes de ansiedad (40).

Con respecto al papel de la depresión en el suicidio, se ha propuesto que los deprimidos tendrían tasas de suicidio superiores a los sujetos sin esta afectación, estimándose que más del 50\% de los suicidios se deben a la depresión (27). En la tentativa de suicidio la depresión juega un papel determinante y afecta principalmente a los adolescentes $(7,40,41)$. En Colombia, de acuerdo a estudios realizados por Toro y colaboradores, el riesgo de suicidio en los adolescentes que manifestaron algún indicador de depresión equivaldría a 4.3 veces el de los adolescentes no depresivos (41).

Para finalizar con los factores de orden personal asociados con las conductas suicidas, algunos estudios indican que es posible que los homosexuales tengan un mayor riesgo suicida, sin embargo, aún no se han llevado a cabo investigaciones con suficiente validez para demostrarlo. Así mismo, en estos estudios los factores que se postulan para justificar el mayor riesgo de ideación suicida, intento suicida y suicidio consumado, son las dificultades para construir la identidad sexual, la disconformidad con el género, historia de abuso físico y/o sexual, bajo nivel de soporte social y abuso de sustancias psicoactivas, alcohol o tabaco $(19,40-44)$.

\section{Factores familiares asociados con la aparición de conductas suicidas}

Dentro de los factores familiares asociados con la aparición de conductas suicidas en adolescentes, se ha investigado la dinámica del funcionamiento familiar como un aspecto determinante. Así, la presencia de comunicación familiar conflictiva, la pobre cercanía afectiva, los altos niveles de control parental, la inestabilidad en la estructura familiar, la historia familiar de conducta suicida y la presencia de violencia intrafamiliar, son factores que contribuyen a limitar la activa y adecuada participación social del adolescente y que dificultan la satisfacción de sus necesidades más básicas $(12,24,30,31,45)$. De igual forma, se han descrito factores como la negligencia y la crianza en hogares monoparentales como otros posibles potenciadores del intento suicida en los jóvenes $(45,46)$.

En relación con la violencia intrafamiliar, se puede decir que la presencia de violencia doméstica bien sea física, verbal o sexual, puede ser un grave condicionante de conductas suicidas $(39,40)$. Un ejemplo de ello es la asociación encontrada entre abuso sexual en la infancia e ideación suicida. El antecedente de abuso sexual ha sido asociado positivamente a la ideación e intento de suicidio, debido probablemente a que el abuso sexual afecta la salud física y psicológica, con resultados como depresión o algunas conductas sexuales de riesgo, que a su vez pueden ser mediadoras de la conducta suicida en adolescentes (20,39). Con referencia a la violencia física, esta forma de agresión puede ocasionar también conducta suicida y, eventualmente la consumación de suicidio $(7,45,47)$. Así, el predominio del maltrato físico y psicológico puede afectar la adecuada comunicación entre los integrantes de la familia e incrementar la ideación suicida en los adolescentes (48).

Es posible también que el maltrato verbal, proveniente especialmente de los cuidadores o progenitores, ya sea en forma de ofensas, descalificaciones, generación de culpa o de críticas hirientes, incida directamente en la presencia de baja autoestima de los jóvenes, lo cual puede derivar a su vez en conductas autodestructivas. En este sentido, se ha señalado que la presencia de vínculos conflictivos entre padres e hijos, así como de baja adaptación de la dinámica familiar ante los cambios que se dan durante la adolescencia, conllevan un mayor riesgo para la aparición de ideación e intento suicida $(12,18,49,50)$. De acuerdo con Toro y cols para los adolescentes que perciben disfunción familiar en su hogar, evidenciada en relaciones conflictivas y poco cálidas, el riesgo de suicidio sería dos veces con relación al de los adolescentes que consideran a su familia funcional (41).

Teniendo en cuenta lo antes mencionado, algunos investigadores han discutido sobre cuáles pueden ser las funciones esenciales de la familia, exponiendo por ejemplo, que las principales características de una familia funcional son las jerarquías claras, los límites claros, los roles definidos, la comunicación abierta y explícita, la afectividad y cooperación entre los miembros y la capacidad de adaptación al cambio. Cuando esto no ocurre se puede generar en los adolescentes conductas suicidas que van desde la ideación hasta el acto consumado $(41,49)$.

Por lo anterior, la percepción que presente el joven en cuanto a escasez de apoyo familiar o en general de conflictos intrafamiliares, puede determinar que sea más vulnerable a concebir conductas suicidas $(22,47)$. Diversos estudios han señalado que los conflictos familiares representan el factor de riesgo de más alta prevalencia en población depresiva puesto que si el adolescente presenta un trastorno mental subyacente este puede percibir un mayor número de eventos como estresantes $(40,49)$. Según Salirrosas y Saavedra, los estilos de crianza donde existe "rechazo, sobreprotección y favoritismo, podrian estar asociados con la presencia de cuadros depresivos asociados a otra patología psiquiátrica y con ideas o actos suicidas, que podrían complicar la evolución del cuadro clínico" (46). Además, aunque la depresión por sí misma es un factor de riesgo entre los adolescentes con conducta suicida, las dificultades en las relaciones familiares le otorgan una contribución significativa independiente a este riesgo $(38,45)$.

El manejo que padres y otros familiares den a las diferentes situaciones influye en la presencia de psicopatologías en los adolescentes sobre lo cual incide también la utilización de 
métodos correctivos inadecuados, que, en lugar de modificar la conducta inapropiada, pueden reforzarla o hacer que creen comportamientos rebeldes hacia la autoridad de los padres $(5,51)$.

Entre los conflictos que se producen durante la crianza, las frecuentes agresiones por parte de sus tutores, con el consecuente efecto de miedo y sentimiento contradictorio, son factores potencialmente psicopatógenos, que pueden generar ideación e intento suicida, debido a la impulsividad propia de los afectados (21). El comportamiento autodestructivo se convierte entonces en un medio de comunicación de sentimientos, frustraciones o necesidades que el adolescente no logra expresar de otra manera (5).

La carencia de calidez familiar, la falta de comunicación con los padres y las continuas discordias familiares hacen que existan pocas posibilidades para el aprendizaje de adecuados métodos de resolución de problemas (45). Lo mencionado anteriormente es coherente con lo expresado por otros autores que sostienen que los adolescentes con conductas suicidas carecen de apoyo emocional por parte de sus familias $(7,45)$.

Por otro lado, se ha encontrado que un factor de riesgo para la aparición de conductas suicidas en adolescentes es la presencia de familias monoparentales, especialmente aquellas con ausencia de figura paterna (46). Así mismo, algunos estudios muestran asociación entre el intento suicida y las alteraciones en la dinámica de la pareja (51). Probablemente, no sea la estructura familiar, sino la calidad de las relaciones familiares el factor de riesgo que influye en la conducta suicida del niño y adolescente temprano $(7,45)$.

Por otra parte, la relación entre ideación suicida adolescente y el antecedente de intento suicida de un familiar ha sido investigada, llegando a determinarse que posiblemente en estas familias podría presentarse una forma de aprendizaje por imitación, proporcionando un modelo de afrontamiento ante complicaciones emocionales o de angustia (50). En una investigación adelantada por Cortés se encontró que la presencia de antecedentes personales y familiares de intento suicida incrementa el riesgo de ejecutar el suicidio; esto es coherente con lo reportado por diversos autores $(17,19,46$, 47, 51).

\section{Factores sociales para el desarrollo de conductas suicidas}

El contexto social donde se desenvuelve el joven adolescente puede influir en gran medida en la aparición de conductas suicidas. Así, la exposición a sucesos sociales relacionados con el desplazamiento, la discriminación social, la exposición a contenidos de información transmitida a través de los diferentes medios de comunicación y la ausencia de redes de apoyo social pueden ser factores de riesgo para el desarrollo de conductas suicidas (52).
En Colombia la discriminación social, el secuestro, la tortura y las heridas de guerra son condiciones que, de manera general, conducen a situaciones de estrés en los adolescentes que favorecen el desarrollo de comportamientos autodestructivos tales como el intento de suicidio y el suicidio consumado. Así mismo, existen otras situaciones consideradas como factores de riesgo para la ideación suicida y otras conductas suicidas en adolescentes tales como las cuestiones de identidad relativas a orientación sexual (18). Debido a la exclusión social, las personas sexualmente distintas a menudo son víctimas de violencia que no les permite el pleno ejercicio de sus derechos sexuales y reproductivos. Esta discriminación afecta la autoestima del adolescente y lo conduce al riesgo de experimentar ansiedad, victimización, persecución, abuso de alcohol, consumo de sustancias psicoactivas, depresión, etc. (53).

De acuerdo con diversos estudios a nivel mundial, se puede afirmar que hay evidencia de que las personas sexualmente distintas tienen un mayor riesgo de presentar conductas suicidas, debido a que frecuentemente sufren mayor rechazo, hostilidad, violencia física e intimidación por la valoración social negativa de su orientación sexual (54). Se ha estimado que la población LGB (lesbianas, gays y bisexuales) tiene un mayor riesgo de suicidio con una asociación positiva entre ser LGB y tener ideación suicida $(55,56)$.

Otro factor que ha sido analizado es la exposición a ciertos contenidos de la información transmitida a través de los diferentes medios de comunicación. En la actualidad, los medios de comunicación ejercen una gran influencia sobre las actitudes, creencias y comportamientos de la comunidad y en especial los adolescentes, quienes son los que están más expuestos a las tecnologías (57). Por lo tanto, es posible que los adolescentes adquieran ciertos comportamientos que van ligados a imitar algunos de los fenómenos que observan en los medios de comunicación, entre los cuales se muestran la ideación suicida, el intento suicida y el suicidio consumado. Este fenómeno recibe el nombre de efecto de imitación o efecto Werther y sucede debido a la influencia de información general mostrada en estos medios (novelas, libros y redes sociales) (58).

En los adolescentes es importante destacar un factor que se ha denominado efecto de la identificación. Según este planteamiento, una conducta suicida puede precipitar otros intentos en un grupo de adolescentes con características similares, lo que se ha descrito también como suicidio por imitación. Un ejemplo de este efecto fue el que se evidenció en el año 1774 , luego de que se publicara la novela de J. W. Goethe "Las cuitas del joven Werther" en la cual el protagonista se quitaba la vida; luego de esta publicación, se notó un aumento en la tasa de suicidio en los jóvenes, quienes, como prueba de la influencia de lo mencionado en el texto, dejaban abierto el libro en la página de este suceso o vestían igual al protagonista de la novela (21). 
Adicionalmente, los adolescentes que consumen sustancias psicoactivas, que presentan trastornos de ansiedad o que sufren aislamiento social, tienen mayor riesgo de cometer suicidio por imitación(21).

Por otro lado, según la OMS, también se ha podido inferir que la difusión de suicidios no ficticios por parte de los medios de comunicación incrementa de manera significativa el número de suicidios en la población expuesta a esta información (1). Dicha influencia es más clara en poblaciones susceptibles como adolescentes y adultos jóvenes, en quienes este tipo de información puede inducir o promover conductas suicidas. Así mismo, se ha podido demostrar que existe una mayor incidencia del suicidio por imitación en individuos con diagnósticos psiquiátricos, del espectro depresivo, con antecedente del maltrato en la infancia, inclusive sin antecedentes familiares de conductas suicidas (21).

Para finalizar, un factor adicional que vale la pena mencionar como determinante para el desarrollo de conductas suicidas es la ausencia de redes de apoyo social. Así, los individuos que experimentan aislamiento en su vida son más vulnerables al suicidio que los que tienen vínculos sociales fuertes con otras personas (5,59-61). Si adicionalmente, las personas con pocos vínculos sufren una pérdida o algún otro tipo de conflicto, el riesgo aumenta pues es difícil el acceso a redes sociales de apoyo hacia sí mismo. El individuo percibe la sensación de que no es necesario, significativo o útil dentro del grupo social en el que se desenvuelve y a su vez, desarrolla depresión y baja autoestima y su aislamiento social reduce las posibilidades de recibir ayuda y evitar atentar contra su vida para resolver la situación(61).
En la Tabla 1 se resumen los factores personales, familiares y sociales más relevantes, relacionados con la aparición de conductas suicidas en adolescentes.

\section{Conclusiones}

El suicidio en los adolescentes es un fenómeno multicausal, relacionado con una serie de factores de orden personal, familiar y social. Así, la aparición de conductas suicidas (ideación suicida, intento suicida o acto suicida consumado), está relacionada con factores como el género, el nivel socioeconómico, el nivel educativo, la salud mental, la dinámica familiar, las redes de apoyo existentes, la inclinación sexual y la exposición a cierto tipo de información, entre otros. La información contenida en la presente revisión podría aportar algunas directrices para el diseño de programas relacionados con este problema de salud pública, más integrales y eficientes, que incluyan la prevención de la aparición de conductas suicidas a través de la oportuna detección y/o manejo de sus posibles factores de riesgo.

\section{Conflicto de intereses}

Los autores declaran no tener conflicto de interés.

\section{Agradecimientos}

A las estudiantes de Psicología de la Universidad Cooperativa de Colombia, Julieth Alejandra Niño Vega,

Tabla 1. Principales factores asociados con la aparición de conductas suicidas

\begin{tabular}{ll}
\hline Dominio & \multicolumn{1}{c}{ Factor } \\
\hline Personal & Edad y género. \\
& Preexistencia de problemas de salud física y mental. \\
& Bajo nivel socioeconómico y educativo. \\
& Consumo de sustancias psicoactivas. \\
\hline Familiar & Problemas en la comunicación. \\
& Pobre cercanía afectiva. \\
& Inestabilidad en la estructura familiar, disfunción familiar, familias \\
& monoparentales. \\
& Excesivos niveles de control parenteral. \\
& Antecedentes familiares de conducta suicida. \\
& Violencia intrafamiliar. \\
\hline Social & Discriminación social. \\
& Carencia de redes de apoyo social. \\
& Efecto de imitación - efecto Werther.
\end{tabular}

Fuente: Elaboración de los autores basado en los aportes de los diferentes autores citados en el manuscrito. 
Romy Margguie Marín Hurtado, Yulie Andrea López Fonseca y Yina Esther Hernández Carrillo por sus valiosos aportes durante la búsqueda, análisis y sistematización de los documentos revisados.

\section{Referencias}

1. Organización Mundial de la Salud. Prevención del suicidio: un imperativo global. [Internet]. 2014. [citado

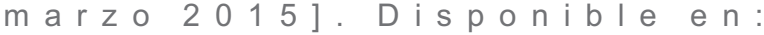
http://www.who.int/mental_health/suicideprevention/world_report_2014/es/.

2. Soler P, Gascón J. Recomendaciones terapéuticas en los trastornos mentales. Tercera edición. Cataluña: ARS Médica; 2010.

3. Moreno C, Diaz C, Soler R, Brossard M, Carbonell I. Caracterización epidemiológica de la conducta suicida en la provincia de Santiago de Cuba. Medisan. 2012; 16(2):205-10

4. Bella M, Fernández R, Willington J. Identificación de factores de riesgo en intentos de suicidio en niños y adolescentes. Rev Argent Salud Pública. 2010; 1(3):249.

5. Caycedo A, Arenas M, Benítez M, Cavanzo P, Leal G, Guzmán Y. Características psicosociales y familiares relacionadas con intento de suicidio en una población adolescente en Bogotá. Pers Bioét. 2010; 14(2):205-13.

6. Rodriguez J, Medina O, Cardona D. Caracterización del suicidio en el departamento de Risaralda, Colombia, 2005-2010. Rev Fac Med. 2013; 61(1):9-16.

7. Hernández A, González I, López Y. Factores de riesgo relacionados con la conducta suicida en la infancia y adolescencia. Medisan. 2013; 17(12):9027-35.

8. Cifuentes S. Comportamiento del suicidio, Colombia 2013. Instituto Nacional de Medicina Legal y Ciencias Forenses. [Internet]. 2013. [citado junio 2015]. D i s p o n i b l e e n : http://www.medicinalegal.gov.co/documents/20143/495 17/Suicidio.pdf.

9. León S, Lainé D. Conducta suicida en adolescentes. En: V Congreso internacional de la niñez, adolescencia y familia. [Internet]. 2013. [citado junio 2015]. Disponible en: http://www.codajic.org/ sites/www.codajic.org/files/ $20 \% 20$ - \% 20 C O N D U C T \% 20 S UICIDA \% $20 \% 20 E N \% 20 A D O L E S C E N T E S . . p d f$

10. Cañón S. Factores de riesgo asociados a conductas suicidas en niños y adolescentes. Archivos de Medicina. 2011; 11(1):62-7.

11. Valdivia M, Silva D, Sanhueza F, Cova F, Melipillán R. Prevalencia de intento de suicidio adolescente y factores de riesgo asociados en una comuna rural de la provincia de Concepción. Rev Med Chil. 2015; 143(3):320-28. Disponible en: http://dx.doi.org/10.4067/S003498872015000300006.

12. Gould M, Fisher P, Parides M, Flory M, Shaffer D. Psychosocial risk factors of child and adolescent completed suicide. Arch Gen Psychiatry. 1996; 53(12):1155-62. Disponible en: https://doi.org/10.1001/ archpsyc.1996.01830120095016.

13. Medina O, Cardona D, Rátiva J. Caracterización del suicidio en Armenia-Colombia, 2004 - 2008. Orbis. 2010; 15(5):76-102.

14. Blandon O, Medina O, Peláez M. Comportamiento de los suicidios en el Urabá Antioqueño, Colombia, 2000-2010.
Rev. Investigaciones Andina. 2015; 17(30):1166-78

15. Peña L, Casas L, Padilla M, Gómez T, Gallardo M. Comportamiento del intento suicida en un grupo de adolescentes y jóvenes. Rev Cub Med Mil. 2002; 31(3):182-87.

16. Brent D, Baugher M, Bridge J, Chen T, Chiappetta L. Age and sex related risk factors for adolescent suicide. J Am Acad Child Adolesc Psychiatry. 1999; 38(12):14971505. Disponible en: https://doi.org/10.1097/00004583199912000-00010.

17. Bella M, Fernández R, Willington J. Intento de suicidio en niños y adolescentes: depresión y trastorno de conducta disocial como patologías más frecuentes. Arch Argent Pediatr. 2010; 108(2):124-29.

18. Cervantes W, Melo E. El suicidio en los adolescentes: un problema en crecimiento. Duazary. 2008; 5(2):148-54.

19. Vega M, Blasco H, Baca E, Diaz C. El suicidio. Salud Ment. 2002; 2(4):1-15.

20. Noa J, Miranda M. Factores de riesgo de intento suicida en adolescentes. Medisan. 2010;14(3):353-59.

21. Sánchez R, Guzmán Y, Cáceres H. Estudio de la imitación como factor de riesgo para ideación suicida en estudiantes universitarios adolescentes. Rev Colomb Psiquiatr. 2005; 34(1):12-25.

22. González, Ramos L, Caballero M, Wagner F. Correlatos psicosociales de depresión, ideación e intento suicida en adolescentes mexicanos. Psicothema. 2003; 15(4):52432.

23. Bridge J, Goldstein T, Brent D. Adolescent suicide and suicidal behavior. J Child Psychol Psychiatry. 2006; 47(3-4):372-94. Disponible en: https://doi.org/10.1111/ j.1469-7610.2006.01615.x.

24. Guibert W, Del Cueto E. Factores psicosociales de riesgo de la conducta suicida. Rev Cubana Med Gen Integr. 2003; 19(5):125-35.

25. Mangnall J, Yurkovich E. A literature review of deliberate self harm. Perspect Psychiatr Care. 2008; 44(3):175-84. Disponible en: https://doi.org/10.1111/j.17446163.2008.00172.x.

26. Renaud G, Berlim M, McGirr A, Tousignant M, Turecki G. Current psychiatric morbidity, aggression/impulsivity and personality dimensions in child and adolescent suicide: a case control study. J Affect Disord. 2008; 105(1-3):22128 D i s p o n i b le e n : https://doi.org/10.1016/j.jad.2007.05.013.

27. Pavez P, Santander N, Carranza J, Vera P. Factores de riesgo familiares asociados a la conducta suicida en adolescentes con trastorno depresivo. Rev Méd Chile. 2009 ; $137(2): 226-33$. Disponible en: http://dx.doi.org/10.4067/S0034-98872009000200006.

28. Nock M, Joiner T, Gordon K, Lloyd-Richardson E, Prinstein M. Non suicidal self-injury among adolescents: diagnostic correlates and relation to suicide attempts. Psychiat Res. 2006; 144(1):65-72. Disponible en https://doi.org/10.1016/j.psychres.2006.05.010.

29. Owens D, Horrocks J, House A. Fatal and non-fatal repetition of self-harm. $\mathrm{Br} \mathrm{J}$ Psychiatry. 2002; 181(3):193-99. Disponible en: https://doi.org/10.1192/ bjp.181.3.193.

30. Wagman I, Ireland M, Resnich M. Adolescent suicide attempts: risk and protectors. Pediatrics. 2001 $107(3): 485-93$. D i s p o n i ble e n : https://doi.org/10.1542/peds.107.3.485.

31. Pelkonen M, Marttunen M. Child and adolescent suicide: epidemiology, risk factors and approaches to prevention. Paediatr Drugs. 2003; 5(4):243-65. 
32. Stanley B, Winchel R, Molcho A, Simeon D, Stanley M. Suicide and the self-harm continuum: phenomenological and biochemical evidence. Int Rev Psychiatr. 1992; 4(2):149-55. Disponible en: https://doi.org/10.3109/ 09540269209066312.

33. Kirchner T, Ferrer L, Forns M, Zanin D. Conducta autolesiva e ideación suicida en estudiantes de enseñanza secundaria obligatoria: diferencias de género y relación con estrategias de afrontamiento. Actas Esp Psiquiatr. 2011; 39(4):226-35.

34. Hagnell O, Lanke J, Rorsman B, Ojesjo L. Are we entering an age of melancholy? Depressive illness in a prospective epidemiological study over 25 years: the Lundby study. Psychol Med. 1982; 12(2):279-89. Disponible en: https://doi.org/10.1017/ S0033291700046614.

35. FernándezA. Claves de la depresión. Segunda edición. Madrid: Cooperación Editorial 2001.

36. Suyemoto K. The functions of self-mutilation. Clin Psychology Rev. 1998; 18(5):531-54. Disponible en: https://doi.org/10.1016/S0272-7358(97)00105-0.

37. Cortés A, Aguilar J, Suárez R, Rodríguez E, Durán J. Factores de riesgo asociados con el intento suicida y criterios sobre lo ocurrido en adolescentes. Rev Cubana Med Gen Integr. 2011; 27(1):33-41.

38. Larraguibel M, González P, Martínez V, Valenzuela R. Factores de riesgo de la conducta suicida en niños y adolescentes. Rev Chil Pediatr. 2000; 71(3):183-91. Disponible en: http://dx.doi.org/10.4067/S037041062000000300002.

39. Espinoza F, Zepeda V, Bautista V, Hernández C, Newton $\mathrm{O}$, Plasencia G. Violencia doméstica y riesgo de conducta suicida en universitarios adolescentes. Salud Pública Méx. 2010; 52(3):213-19.

40. Mars B, Heron J, Crane C, Hawton K, Kidger J, Lewis G, et al. Differences in risk factors for self-harm with and without suicidal intent: findings from the ALSPAC cohort. J Affect Disord. 2014; 168:407-14. Disponible en: https://doi.org/10.1016/j.jad.2014.07.009.

41. Toro D, Paniagua R, González C, Montoya B. Caracterización de adolescentes escolarizados con riesgo de suicidio, Medellín, 2006. Rev Fac Nac Salud Pública. 2009; 27(3):302-08.

42. Cabra O, Infante D, Sossa F. El suicidio y los factores de riesgo asociados en niños y adolescentes. Revista Médica Sanitas 2010;13(2)28-35.

43. Hallfors K, Waller M, Ford C, Halpern C, Brodish P, Iritani B. Adolescent depression and suicide risk: Association with sex and drug behavior. Am J Prev Med. 2004; 27(3):224-31. Disponible en: https://doi.org/10.1016/ j.amepre.2004.06.001.

44. Russell S, Joyner K. Adolescent sexual orientation and suicide risk: evidence from a national study. Am J Public Health. 2001; 91(8):1276-81. Disponible en: https://doi.org/10.2105/AJPH.91.8.1276.

45. Guibert W, Torres N. Intento suicida y funcionamiento familiar. Rev Cubana Med Gen Integr. 2001; 17(5):45260.
46. Salirrosas C, Saavedra J. Percepción de algunos estilos de crianza y el episodio depresivo en el adulto. Rev Neuropsiquiatr. 2014; 77(3):160-67.

47. Pérez S. El suicidio, comportamiento y prevención. Rev Cubana Med Gen Integr. 1999; 15(2):196-217.

48. Gould M, Greenber T, Velting D, Shaffer D. Youth suicide risk and preventive interventions: a review of the past 10 years. J Am Acad Child Adolesc Psychiatry. 2003; 42(4):386-405. Disponible en: https://doi.org/ 10.1097/01.CHI.0000046821.95464.CF.

49. Andrade J. Aspectos psicosociales del comportamiento suicida en adolescentes. Revista Electrónica de Psicología Iztacala. 2012; 15(2):688-721.

50. García J, Quintanilla R, Sánchez L. Morfín T, Cruz J. Consenso cultural sobre el intento de suicidio en adolescentes. Rev colomb psicol. 2011; 20(2):167-79.

51. Valadez I, Amezcua R, Quintanilla R, González N. Familia e intento suicida en el adolescente de educación media superior. Archivos en Medicina Familiar. 2005; 7(3):69-78.

52. Tuesca R, Navarro E. Factores de riesgo asociados al suicidio e intento de suicidio. Salud Uninorte. 2003; $17: 19-28$.

53. Pineda C. Etiología social del riesgo de suicidio en adolescentes y jóvenes lesbianas, gay y bisexuales: una revisión. Psicogente. 2013;16(29):218-34.

54. Ortiz L. Influencia de la opresión internalizada sobre la salud mental de bisexuales, lesbianas y homosexuales de la ciudad de méxico. Salud Ment. 2005; 28(4):49-65.

55. King M, Semlyen J, See S, Killaspy H, Osborne D, Popelyuk D, et al. Asystematic review of mental disorder, suicide, and deliberate self harm in lesbian, gay and bisexual people. BMC Psychiatry. 2008; 70(8):1-17. Disponible en: https://doi.org/10.1186/1471-244X-8-70.

56. Ralfman J, Moscoe E, Austin B, MacConnell M. Difference-in-differences analysis of the association between state same-sex marriage policies and adolescent suicide attempts. JAMA Pediatr. 2017; 171(4):350-56. Disponible en: https://doi.org/10.1001/ jamapediatrics.2016.4529.

57. Herrera R, Ures M, Martínez J. El tratamiento del suicidio en la prensa española: ¿efecto werther o efecto papageno?. Rev Asoc Esp Neuropsiq. 2015; 35(125):123-34. Disponible en: http://dx.doi.org/ 10.4321/S0211-57352015000100009.

58. Villagómez R, Balcázar A, Paz R. Suicidio en jóvenes. Rev Fac Med UNAM. 2005; 48(2):54-57.

59. Di Rico E, Paternain N, Portillo N, Galarza A. Análisis de la relación entre factores psicosociales y riesgo suicida en adolescentes de la ciudad de Necochea. Perspectivas en Psicología. 2016; 13(2):95-06.

60 . Shain B. Suicide and suicide attempts in adolescents. Pediatrics. 2016; 138(1):1-11. Disponible en: https://doi.org/10.1542/peds.2016-1420.

61. Siabato E, Forero I, Salamanca Y. Asociación entre depresión e ideación suicida en un grupo de adolescents colombianos. Pensam psicol. 2017; 15(1):51-61. Disponible en: http://dx.doi.org/10.11144/ Javerianacali. PPSI15-1.ADIS. 